\title{
Analyzing Vehicle Fuel Saving Opportunities through Intelligent Driver Feedback
}

\author{
Jeffrey Gonder, Matthew Earleywine and Witt Sparks \\ National Renewable Energy Laboratory
}

\begin{abstract}
While it is well known that "MPG will vary" based on how one drives, little independent research exists on the aggregate fuel savings potential of improving driver efficiency and on the best ways to motivate driver behavior changes. This paper finds that reasonable driving style changes could deliver significant national petroleum savings, but that current feedback approaches may be insufficient to convince many people to adopt efficient driving habits. To quantify the outer bound fuel savings for drive cycle modification, the project examines completely eliminating stop-and-go driving plus unnecessary idling, and adjusting acceleration rates and cruising speeds to ideal levels. Even without changing the vehicle powertrain, such extreme adjustments result in dramatic fuel savings of over $30 \%$, but would in reality only be achievable through automated control of vehicles and traffic flow. Considering the effects of real-world driving conditions, efficient driving behaviors could reduce fuel use by $20 \%$ on aggressively driven cycles and by $5-10 \%$ on more moderately driven trips.
\end{abstract}

To evaluate potential receptiveness to changing driving habits, the project team conducted a literature survey of driver behavior influences and observed pertinent factors from on-road experiments with different driving styles. This effort highlighted important driver influences such as surrounding vehicle behavior, anxiety over trying to get somewhere quickly, and the power/torque available from the vehicle. Existing feedback approaches often effectively deliver efficiency information and instruction, but do not always do so in an easy way that avoids unintended consequences and helps trump other driving behavior influences. Based on these findings the report details three recommendations for maximizing fuel savings from potential drive cycle improvement: (1) Leverage applications with enhanced incentives, (2) Use an approach that makes it easy and is widely-deployable to motivated drivers, and (3) Utilize connected vehicle and automation technologies to achieve large and widespread efficiency improvements.

\section{INTRODUCTION}

Data show that the reported fuel economy for a given vehicle can vary by plus or minus $20 \%$ across a large population of drivers [1]. Some of this variation results from differences in temperatures and road conditions encountered, but differing driver behavior also accounts for some of this spread. It stands to reason that if drivers universally adopted more efficient driving habits, then the spread in fuel economy results for any given vehicle would shift to higher values. The U.S. Department of Energy (DOE) asked the National Renewable Energy Laboratory (NREL) to evaluate the potential fuel saving opportunity from implementing driver feedback techniques and to begin evaluating which techniques may deliver the greatest aggregate fuel savings. This paper summarizes NREL's findings.

\section{APPROACH}

NREL divided the driver feedback investigation into three separate tasks: (1) quantifying the fuel saving opportunities from drive cycle modification; (2) identifying factors that influence drivers' receptiveness to adopt efficient behaviors; and (3) assessing various driver feedback approaches. NREL performed both simulations and experiments for the first task, and leveraged a conventional vehicle model from a previous study for the simulation effort [2]]. The model possessed similar design and fuel economy characteristics as a midsize Chevrolet Malibu [1]. Note that this study primarily focused on conventional (rather than hybrid) vehicle performance because of DOE's interest in what driver feedback technology could do for the existing U.S. vehicle fleet, which primarily consists of conventional powertrain vehicles. 
Table 1. Simulated conventional vehicle fuel savings for ideal vs. real-world cycles

\begin{tabular}{|c|c|c|c|c|c|c|c|c|}
\hline \multirow[b]{2}{*}{ Cycle Name } & \multirow[b]{2}{*}{$\mathrm{KI}(1 / \mathrm{mi})$} & \multirow[b]{2}{*}{ Distance (mi) } & \multicolumn{2}{|c|}{ Fuel Economy (mpg) } & \multirow[b]{2}{*}{$\%$ Increase } & \multicolumn{2}{|c|}{ Gallons Used } & \multirow[b]{2}{*}{$\%$ Decrease } \\
\hline & & & Real World & Improved & & Real World & Improved & \\
\hline 20122 & 4.55 & 1.3 & 17.0 & 39.5 & $132 \%$ & 0.08 & 0.03 & $57 \%$ \\
\hline $2145 \_1$ & 1.03 & 11.2 & 31.0 & 49.8 & $61 \%$ & 0.36 & 0.22 & $38 \%$ \\
\hline 4234_1 & 0.87 & 58.7 & 30.5 & 52.7 & $73 \%$ & 1.92 & 1.11 & $42 \%$ \\
\hline $2032 \_2$ & 0.24 & 57.8 & 35.2 & 52.7 & $50 \%$ & 1.64 & 1.10 & $33 \%$ \\
\hline 4171_1 & 0.16 & 109.4 & 32.7 & 53.1 & $62 \%$ & 3.34 & 2.06 & $38 \%$ \\
\hline
\end{tabular}

The vehicle model was simulated over a variety of drive cycle conditions to observe the fuel efficiency impact. The analyzed cycles included those from a large set of real-world travel data collected in 2006 with global positioning system (GPS) devices as part of a study by the Texas Transportation Institute and the Texas Department of Transportation [3] . NREL examined the efficiency benefit from applying hypothetical modifications to the GPS drive cycles, and also observed the relative prevalence of inefficient vs. already efficient driving characteristics within the data set.

To confirm what reasonable degree of drive cycle modifications could be implemented on-road, NREL researchers variously drove an instrumented vehicle over repetitions of a "city" route with a large number of stoplights and a "highway" route with primarily interstate driving. The routes were repeated by two drivers using three different driving styles: "normal" (as the driver would normally drive); "energy conscious" (making a best effort to implement efficiency techniques while still driving safely); and "aggressive" (defined as how the driver might behave if running late to get somewhere while still doing so safely).

To evaluate driver receptiveness to feedback, NREL examined why drivers chose particular behaviors and what factors may serve as barriers to behavior change. These efforts included: (1) a literature search of driving behavior influences and issues, and (2) documenting the motivations behind driving decisions from the on-road driving experiments.

To complete the final assessment task, NREL tested several devices and evaluated various feedback approaches in the context of the first two tasks' findings-i.e., could the approach result in quantitative fuel savings, and are people likely to start driving more efficiently in response to it?

\section{QUANTIFYING FUEL-SAVING OPPORTUNITIES}

\section{Hypothetical Drive Profile Adjustments}

To quantify the outer boundary fuel savings that could be achieved from drive cycle modification, the authors examined the idealized results of fully optimizing several cycles from the real-world data set mentioned above [ 3$]$. The cycles were selected to reflect a range of kinetic intensity (KI) values. KI represents a ratio of characteristic acceleration to aerodynamic speed and has been shown to be a useful drive cycle classification parameter [4]. The real-world cycles were converted into equivalent "ideal" cycles using the following steps:

1. Calculate the trip distance of each sample trip.

2. Eliminate stop-and-go and idling within each trip.

3. Set the acceleration rate to $3 \mathrm{mph} / \mathrm{s}$.

4. Set the cruising speed to $40 \mathrm{mph}$.

5. Continue cruising at $40 \mathrm{mph}$ until the trip distance is reached.

As summarized in Table 1, the results indicate a fuel savings potential in excess of $30 \%-40 \%$ from full cycle optimization. While these results would not be achievable in reality without full automation of vehicles and traffic control, it is promising to note such significant fuel savings from cycle modifications alone with no changes to the vehicle or powertrain.

Figures 1 and $\underline{2}$ examine the impact of applying a few of the cycle optimization steps in isolation. For instance, the points along the vertical axis in Figure 1 represent the fuel savings benefit solely from eliminating stops in the five sample cycles. This elimination of stop-and-go driving yields the greatest benefit for the high KI cycles (for which acceleration power demands tend to dominate due to a large number of stops in the unmodified cycles). The points to the right of the vertical axis in Figure 1 represent the benefit of eliminating slow-and-go events below the indicated speed in addition to eliminating the stop-and-go, which results in a small additional savings for each cycle. Other simulations conducted as part of this study identified a small fuel savings benefit simply from reducing the rate of acceleration events, but the savings were not nearly as great as from avoiding accelerations and decelerations (accel/decel) altogether. This suggests that driving style improvements should focus on reducing the number of stops in high $\mathrm{KI} /$ city-type cycles, and not just the rate of accelerating out of a stop.

One way for drivers to reduce stop-and slow-and-go driving is to select routes with fewer stops and drive at less congested 


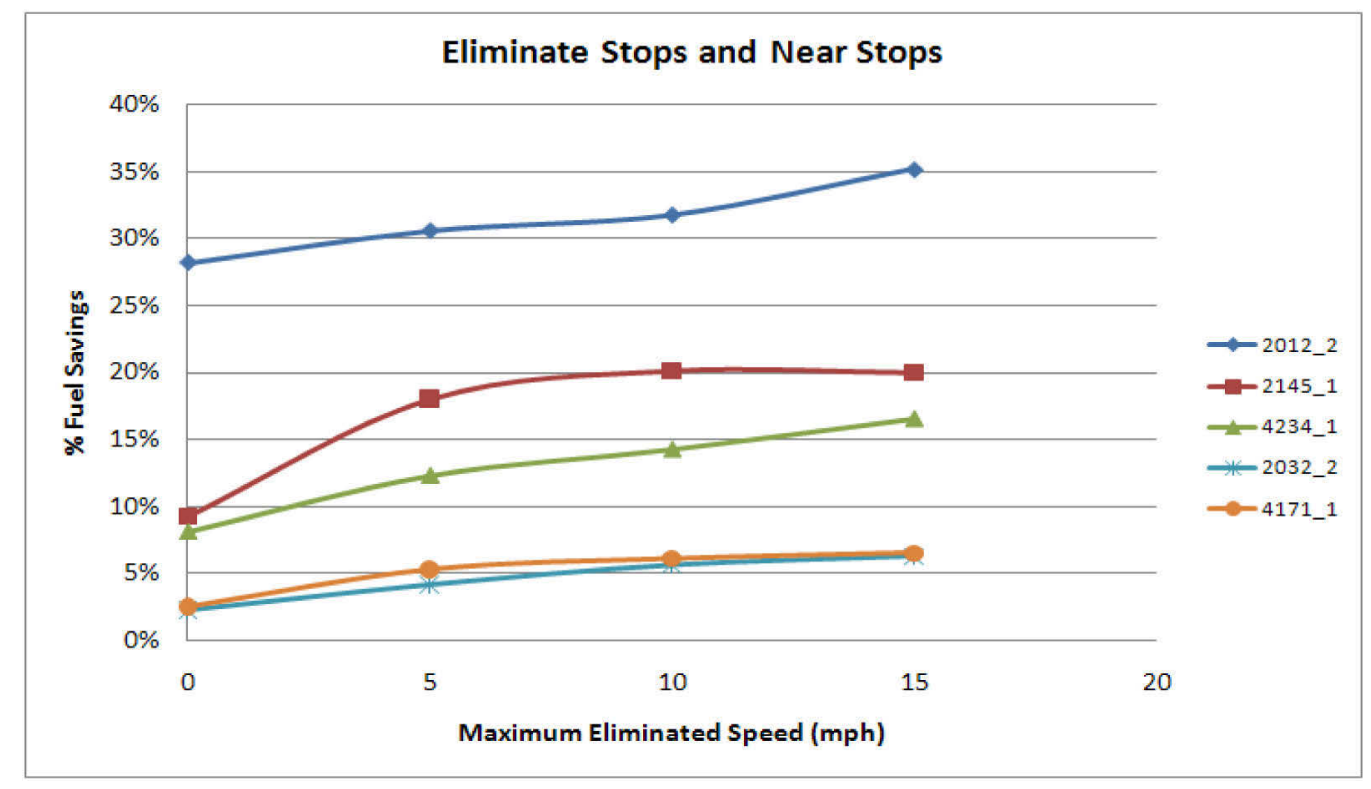

Figure 1. Fuel savings from eliminating stop- and slow-and-go driving

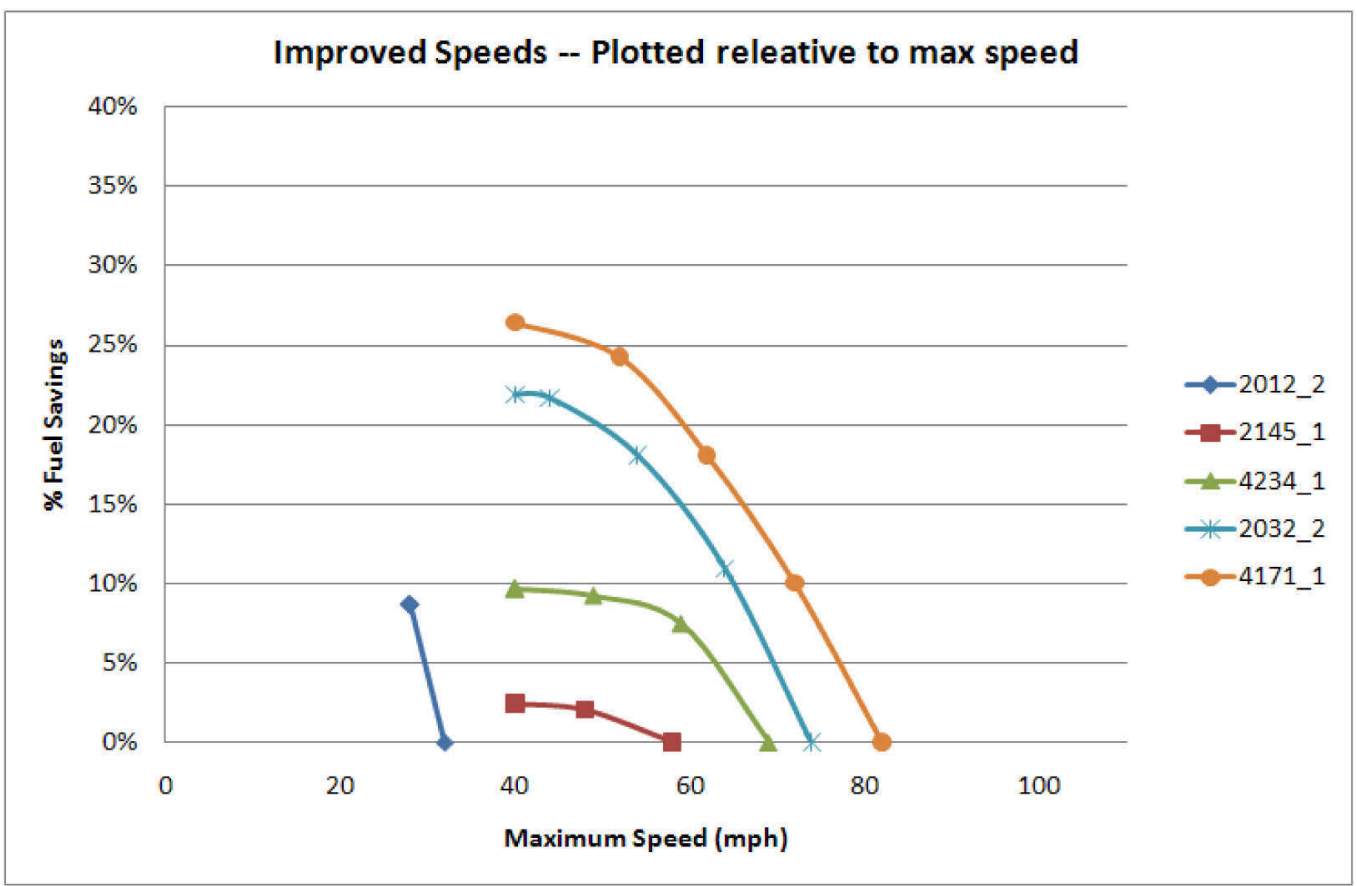

Figure 2. Fuel savings from optimizing vehicle speed

times of day. However, drivers can also reduce stops over the course of a given driving route by watching far enough ahead to anticipate when maintaining speed will result in a stop at a red light or traffic bottleneck. If the driver instead eases off of the gas pedal to start gradually decelerating early, the light could change and the traffic could clear before the driver gets there. Such an approach not only decreases accel/decel rates but also (and more importantly) their frequency of occurrence.
Figure 2 examines the fuel savings from incremental speed optimization and highlights that the largest speed improvement savings result from cycles with the highest driving speeds. These tend to be the cycles with the lowest KI values (where aerodynamic road loads dominate in highwaytype driving). Since aerodynamic drag increases with the square of vehicle velocity, even modest reductions from very high speeds result in significant fuel savings. 


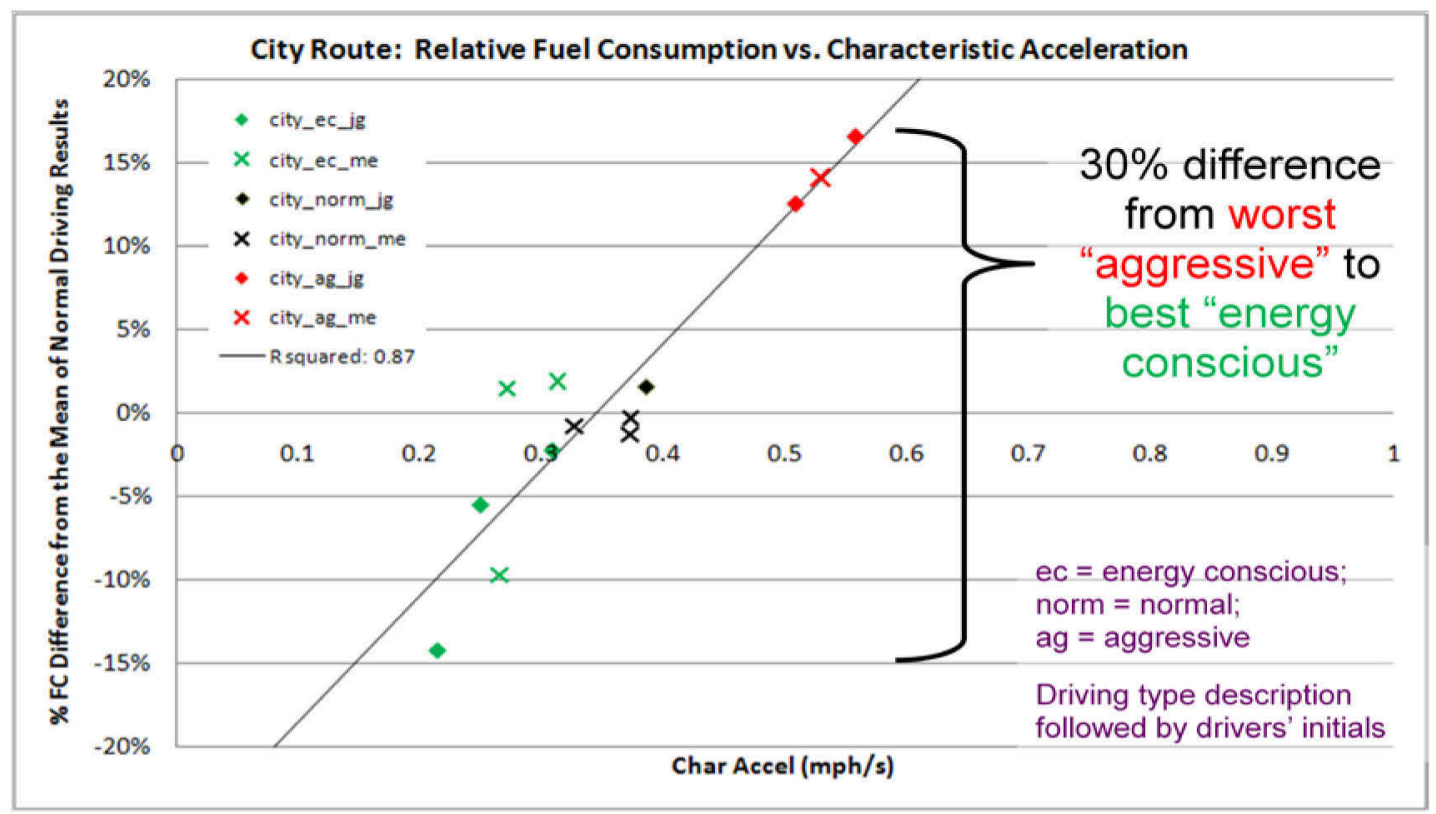

Figure 3. Fuel use comparison for "city" driving experiments

\section{Implementing Efficiency Strategies Over Repeated}

\section{On-Road Routes}

The analysis discussed to this point reflects theoretical savings from targeted cycle improvements that may be difficult to implement on-road. To observe the obstacles to efficient driving first-hand and to perform a sanity check on potential fuel savings over a constant driving route, the project team conducted a series of on-road driving experiments in the Denver area.

The two selected routes (one with city-type driving and one with highway-type driving) were designed to have cycle characterization parameters that fell in the mid range of corresponding parameters from the Texas travel survey onroad cycles [3]. Two drivers (M. Earleywine and J. Gonder) took turns driving the routes. While one person drove, the other sat in the passenger seat to record test observations, including apparent influences on the driver's behavior. A Mercedes C100 test vehicle was used for the driving experiments, and second-by-second data were collected from the vehicle's on-board diagnostic (OBD) port. Chassis dynamometer testing verified the accuracy of the vehicle speed data collected from the OBD port, but unfortunately reliable fuel use data could not be collected. Instead, fuel use estimates were generated by simulating the collected drive profiles using the aforementioned conventional vehicle model (to be consistent with the travel survey data analysis). As described in the Approach section, each route was repeated with three different driving styles.

Figure 3 shows the spread in fuel consumption results for the city route plotted against the characteristic acceleration for each measured profile. The different colors represent different driving behaviors, and the different shapes represent different drivers. The legend provides an abbreviation for the driving type ("ec" for energy conscious, "norm" for normal, or "ag" for aggressive) followed by the initials of the driver for the indicated cycles. Figure 4 provides a similar plot for the highway route, where the relative fuel consumption results are instead plotted against the aerodynamic driving speed for each cycle [ㄴ] .

Both figures show some variability in fuel consumption even when repeating a route with the same driving style. These variations likely result from differences in traffic conditions and stoplight timing from one repetition to the next. Nevertheless, fuel consumption for both routes does seem to trend lower for "energy conscious" as compared to "normal" driving and to trend higher for "aggressive" driving.

Figure 3 shows a $30 \%$ spread between the lowest fuelconsuming energy-conscious repetition and the highest fuelconsuming aggressive repetition of the city route. The apparent correlation between fuel consumption differences and characteristic acceleration is consistent with the earlier finding that reducing accel/decel seems to carry the largest benefit in city-type driving with significant stop- or slow-andgo events.

Figure 4 reveals an approximate $20 \%$ spread between the lowest fuel-consuming energy-conscious repetition and the highest fuel-consuming aggressive repetition of the highway route. The fuel consumption differences for this route seem to roughly correlate with aerodynamic speed, which is again consistent with the earlier findings that reducing high speeds seems to be the dominant factor for improving highway 


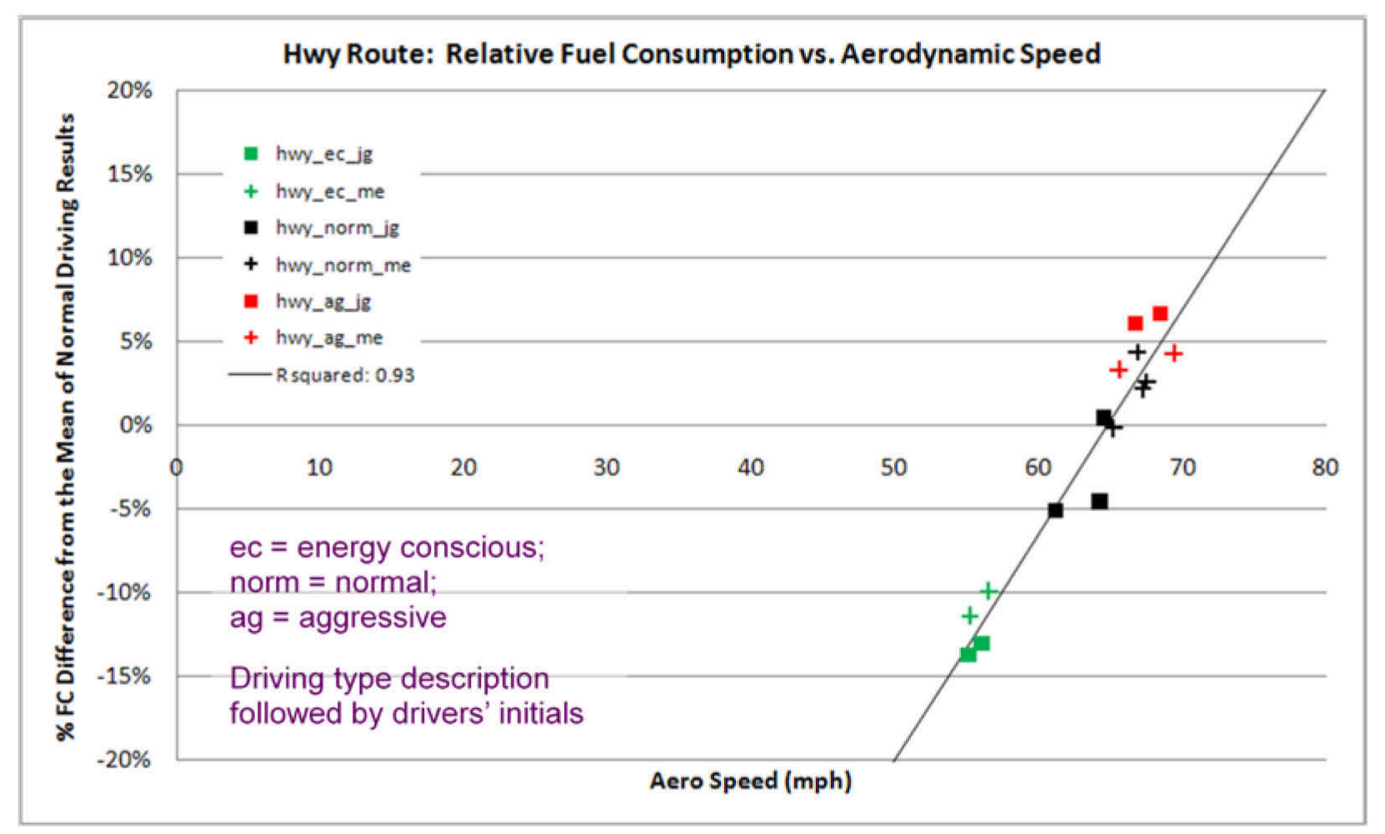

Figure 4. Fuel use comparison for "highway" driving experiments

driving efficiency. While the maximum predicted savings is quite a bit higher for some of the travel survey cycle adjustments, it should be noted that the top speeds for the "aggressive" NREL experiments were much lower than those for the fastest driving vehicles in the travel survey sample.

\section{Prevalence of Inefficient/Sub-optimal Driving}

While the above discussion focused on the fuel saving potential from specific behavior changes, this section considers the prevalence of inefficient behaviors in a large, real-world driving sample. The Texas travel survey data again provide a useful reference for this analysis [ $\underline{3}$ ]. Figure 5 illustrates the simulated fuel consumption results (using the color scale on the right-hand side) for each individual trip in the GPS driving sample that traversed one or more miles. The figure represents each of these nearly 4,000 trips with a small circle and positions it relative to the two axes based on the cycle metrics (aerodynamic speed and characteristic acceleration) that seemed to correlate with fuel efficiency for the on-road driving experiments.

Consistent with the on-road experiment observations for lowspeed city driving, higher characteristic acceleration corresponds with higher fuel consumption in the scatter plot (although as aerodynamic speed drops below $25 \mathrm{mph}$, trip fuel consumption increases even with low characteristic acceleration). For the higher-speed (highway) trips, Figure 5 shows increasing fuel consumption as aerodynamic speed rises above $60 \mathrm{mph}$ (again consistent with the previous analysis results). Note, though, that increased characteristic acceleration in highway trips also results in higher fuel consumption.
The figure highlights that the lowest fuel consuming trips have aerodynamic speeds between roughly $25-60 \mathrm{mph}$ and characteristic accelerations below about $0.3 \mathrm{mph} / \mathrm{s}$. The large number of trips with slightly higher fuel consumption has slightly higher characteristic acceleration, and/or aerodynamic speeds that fall outside of the 25-60 mph range. The small number of very high fuel-consuming trips has characteristic accelerations and/or aerodynamic speeds far from the minimum-consumption operating conditions.

\section{DRIVER RECEPTIVENESS CONSIDERATIONS}

NREL consulted with experts in social science and human factors engineering for advice on what type of feedback drivers would be most receptive to receiving. The experts recommended focusing on the context of those behaviors that could lead to fuel savings if changed. Assessing feedback approaches against the specific context would be more fruitful than trying to identify the "best" specific stimulation type (light, sound, color, text, etc.). To improve contextual understanding of driving behavior influences, the results summarized here are from a literature review and from observations during the on-road driving experiments.

\section{LITERATURE REVIEW OF DRIVING BEHAVIOR INFLUENCES AND ISSUES}

A review of prior research found a number of studies involving fuel economy, driving behavior, and general human behavior as related to energy-saving practices. These findings are summarized below. 


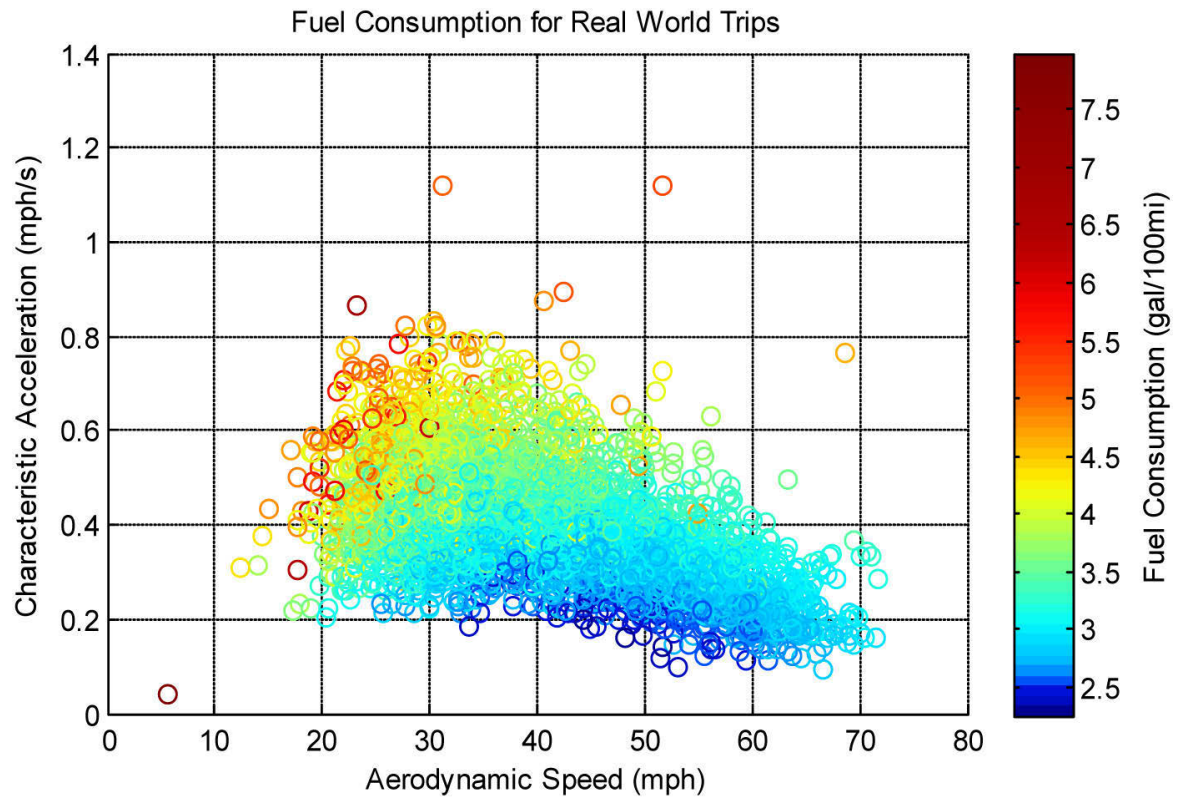

Figure 5. Cycle metrics and simulated fuel consumption for nearly 4,000 real-world trips

\section{Potential for Fuel Savings}

There is broad consensus from prior research that vehicle fuel savings of $10 \%$ are possible through modified driver behavior (a finding also supported by this study). Such savings can be attained through reasonably moderate behavior modification without resorting to extreme "hyper-miling" techniques. One study estimates that if a third of all U.S. drivers adopted ecodriving techniques, it would result in an annual savings of 33 million metric tons of carbon dioxide $\left(\mathrm{CO}_{2}\right)$ and a cost savings of $\$ 7.5-\$ 15$ billion [ㄷ].

Reference [ $\underline{5}$ ] proposes that achieving significant fuel savings through driver behavior changes requires substantial investment in a multi-faceted approach involving:

1. Public education

2. Driver feedback

3. Regulatory actions

4. Economic and policy incentives

5. Social marketing.

\section{Driver Behaviors}

The driving behaviors that affect fuel economy are well understood through existing research and are further supported by this study's findings. Those behaviors include:

1. Speed during highway driving.

2. KI or frequency and intensity of braking and acceleration [].

3. Frequency of stops - faster acceleration is acceptable if it means that a stop is avoided [7].
4. Timing of gear changes [6] -not a focus in the present study due to the current market dominance of automatic transmission vehicles.

5. Time to Collision (TTC) values [6]-metric defined as the following distance from one car to another divided by the speed difference (when the second car is overtaking the first). The study observed that drivers following an efficiency regimen tended to have fewer instances of small TTC values. This metric directly relates to KI: if the driver is trying to reduce KI, he or she will avoid rapidly approaching another vehicle and then braking to avoid collision.

\section{Effect of Social Norms on Driver Behavior}

Reference [ $[\underline{8}$ ] identified two "axes" of norms that influence driver behavior: Legal or regulatory norms, and informal or social norms. The study posits that drivers who violate either or both of those norms are more likely to be involved in accidents because other drivers are unable to predict their actions. This conclusion is supported by evidence that drivers who travel at close to the median speed of traffic on a highway are less likely to be involved in accidents than drivers who tend to drive either faster or slower than that speed. This finding suggests that:

1. Harnessing the natural pressure to conform to surrounding traffic behavior may help a driver feedback approach succeed at getting drivers with very high speeds and accelerations to slow down.

2. A feedback device that encourages behavior outside the social norm for a given situation may encounter driver resistance to deviate from the informal norm.

3. Drivers who do follow feedback advising behavior outside of the norm (for example, driving at a more efficient $55 \mathrm{mph}$ 
on a roadway where the median speed is $70 \mathrm{mph}$ ) may increase their risk of being in an accident.

Other research into consumer behavior related to home energy efficiency indicates that significant and lasting behavior change is possible under the right circumstances. Following a natural disaster that interrupted electricity distribution, the city of Juneau, Alaska, cut its electricity consumption by more than 30 percent. After repairs were completed, energy consumption remained $10 \%$ lower than pre-event levels [9]. Another study reports that providing social comparisons can motivate households to reduce energy consumption [10]. The residential energy consumption data required to make such comparisons are already collected by the local utility. However, since an analogous real-time dataset does not exist for transportation fuel use, it would be more difficult to apply such social comparisons to this domain.

The iPhone driver feedback application Bliss Trek (no longer available) attempted to do this by linking to a user's Twitter account and allowing the user to broadcast driving efficiency scores-in effect creating a competition among users. While this feature was broadcast-only, one could envision that adding the current high score(s) to the application's real-time display would enhance the immediacy of the competition.

\section{Potential for Adoption and Use of Feedback}

\section{Systems}

To assess the impact fuel economy has on vehicle purchase decisions, a research study interviewed 57 households prior to 2007. The study found that most households do not have a realistic grasp of how much money they spend in a week, month or year on fuel. This result indicates that the modest fuel savings associated with driver behavior modifications may be insufficient to incentivize their adoption. The study also found that fuel economy was not named as a concern when purchasing a vehicle among most of the middle-to high-income participants [11].

In a more recent survey [12], Consumer Reports found that prospective vehicle buyers listed fuel economy as a top factor of interest. This survey was conducted in the wake of the 2008 fuel price spike, suggesting a possible temporary increase in concern for fuel economy. In a recent survey in Japan [13], J.D. Power Asia Pacific found improved customer satisfaction with auto dealers who provided advice for maximizing fuel economy when driving. This further supports the thesis that consumers do have some interest in fuel economy with the right encouragement.

Taken together, these studies suggest that although consumers do have an interest in improving fuel economy, that interest is closely tied with fuel prices and perhaps also an emotional response to rapid increases in fuel price. Absent such high or increasing price conditions, potential fuel cost savings alone may be insufficient to motivate behavior change.

A recent California study of the effect of driver feedback on fuel economy included a user survey of background, likelihood, and attitude toward eco-driving. The 20 participants in the survey were concerned about climate change (7.3 on a scale of 10) and likely to adopt eco-driving techniques in the near future (7.4). They were unlikely (3.9) to purchase a driver feedback device, but likely to use one (8.0) if it comes standard with their next vehicle [14].

This research indicates that simply building a driver feedback device and hoping that consumers will purchase and use it will not lead to significant fuel savings overall. There must be some additional incentive or partnership that either offsets the cost of the device or provides some other benefit to incentivize its use.

Other considerations that can impact adoption and use of feedback systems include:

1. A finite time window may exist in which to educate feedback device users. After a few weeks of observing what the device does under different conditions, a driver will likely start paying less and less attention to it.

2. Feedback provided at different time scales and tied in with different events may be useful. For instance, in addition to real-time instruction while driving, cumulative feedback could be provided every few hundred miles when refueling and/or every few thousand miles when getting an oil change.

3. Different solutions may work better for people with low motivation vs. those with high motivation to adopt efficient driving behaviors.

4. Feedback is most effective when it highlights benefits/ savings rather than punishment/penalty, when it helps minimize any negative impacts of the behavior change, and when it specifically relates to a person's own experience/ context (e.g., provides fuel economy relative to that for other drivers of the same vehicle and in the same area).

\section{Design of Driver Feedback Systems}

The simplest driver feedback system, an instantaneous fuel economy gauge, was evaluated in a 1977 study involving 140 test vehicles, half of which were equipped with an instantaneous fuel economy meter. Drivers were tasked with reducing their fuel use over a 12-week period. The study found that meter-equipped vehicles had on average 3\% lower fuel consumption than non-equipped vehicles, but the author reported that this difference was not statistically significant [7].

Instantaneous fuel economy gauges are not especially helpful in urban driving because they do not address specific 
behaviors and because instantaneous fuel economy changes so quickly and significantly when accelerating and decelerating. A gauge by itself also has limited utility in the absence of accompanying instruction or intuitive driver understanding of how to improve fuel economy.

A more recent study of driver feedback systems built into existing vehicles concluded that the most effective systems provided a binary (yes/no) indication of whether or not current behavior is efficient [15]. This study focused only on existing displays, so it provides little insight into which other general design elements are most effective. It also focused on a person's initial ability to interpret a display and did not take into account the display's effectiveness after the driver became accustomed to it.

\section{Potential for Driver Distraction}

A 2007 study examined research into driver distraction caused by a variety of in-car devices, including entertainment systems, navigation systems, and mobile phones [16]. The most relevant research to this study involves the level of driver distraction caused by navigating a vehicle with the aid of a navigation system. This line of research was chosen for comparison because driver feedback systems do not require the driver to provide any information to the device, only to receive and process information from the device.

The review cited research that compared a head-down electronic map, a head-up turn-by-turn guidance display with head-down electronic map, voice guidance with head-down electronic map, and paper map. The study measured driving speed, workload, navigation errors, and reaction time to external events while interacting with the navigation system.

The voice guidance/electronic map system resulted in better performance with lower workload ratings, faster mean speeds, and lower numbers of navigational errors. The study also reports that eyes-off-road times and the level of cognitive effort required to complete a task are indicators of the level of driver distraction.

From this research, the authors conclude that:

1. A voice or audible feedback mechanism may be preferable from a driver distraction point of view because it does not require the driver to look away from the road to take in the information. An audio-only approach may also be more convenient for the user if it eliminates the need to mount an aftermarket display and potentially run wires to it.

2. The information provided should be made as simple to understand as possible to minimize the cognitive effort required to process it.

Reference [6] suggests two approaches to providing feedback: tactical and strategic. Tactical is instantaneous feedback on acceleration, braking, and speed. This, to a large degree, is the approach taken by most of the devices surveyed as part of this study. Strategic feedback analyzes driving behavior over some time period and advises the driver in specific terms on how to improve fuel economy (for example, "let off the accelerator sooner when slowing"). The DriveGain iPhone application provides this type of advice in addition to a visual tactical display.

Because of its real-time nature, it would be difficult to present tactical feedback audibly, but strategic feedback can certainly be presented this way. Reference [ $[\underline{6}]$ did not investigate the effects of these two feedback approaches separately. Future research may be warranted to determine whether a tactical, strategic, or combined approach is best $[\underline{6}$, 7].

In reviewing existing feedback devices, NREL found the cognitive effort required to interpret and understand the visual and audible feedback was not excessive. However, because visual systems do require taking eyes off the road, they should be designed and placed so that information can be pulled off of them with only a quick glance (less than two seconds) and conform to guidelines for mitigating driver distraction potential [17].

\section{FACTORS IMPACTING EFFICIENT DRIVING DECISIONS}

To evaluate the effectiveness of a driver feedback mechanism, it is important to understand how other influences compare with a driver's potential desire to follow the mechanism's advice. To gain a better understanding of these issues, the vehicle passenger during the on-road driving experiments described in the previous section recorded observations about various factors that influenced the driver.

One of the main observations from the on-road driving experiments was that driving at slow speeds and slow accelerations can annoy other drivers on the road. During more than one of the energy-conscious test drives, another driver honked impatiently in response to gradual accelerations. Slow speeds also cause the driver to pay more attention to vehicles behind rather than vehicles ahead since the vehicles ahead were often pulling away and the vehicles behind were tailgating. It was also observed that free-flowing traffic will often drive slightly above the posted speed limit.

Light traffic conditions can make it easier for a motivated driver to implement fuel-efficient techniques by alleviating concerns about holding up the vehicles behind. However, light traffic can discourage a marginally motivated driver from driving efficiently if the other cars on the road speed past on the left. Heavier traffic will increase the number of stops, but can also decrease the magnitude of accelerations and of high speeds. Therefore, heavy traffic has the potential to decrease the fuel consumption of an aggressive driver, 
although it will likely increase the fuel consumption of most vehicles if it forces additional stop-and-go driving.

A given driver may not exhibit the same types of driving behavior all of the time. If a driver is pressed for time and in a hurry, he or she will most likely drive more aggressively. If someone is sightseeing and driving with no specific time frame or destination in mind, he or she will tend to drive and accelerate more slowly, making it easier to drive efficiently.

Relative to driving aggressively, driving energy-efficiently at slower speeds and lower accelerations requires less attention from the driver. An aggressive driver constantly looks ahead for opportunities to change lanes and pass other vehicles, whereas a more fuel-efficient driver can just stay in the right lane and let other vehicles pass him or her.

For powerful vehicles, it can be difficult to maintain the light accelerator pedal pressure required for steady speed cruising. In addition, because a vehicle's purchase price depends greatly on its engine size, owners may be reluctant not to take advantage of the full power capability for which they paid. Fuel efficiency feedback may have to compete with the enjoyment many people have from driving in a sporty manner. Note that over time the vehicle market as a whole has demonstrated consumer preferences for weight and performance rather than for less powerful and slower vehicles (which inherently cost less and have better fuel efficiency [18]).

While considering whether a financial hardship might lead a person to change driving habits, it was noted that other alternatives may be more strongly considered, such as taking public transportation, walking, biking, or carpooling. A driver with an acute need to reduce fuel cost might expect greater savings from such a mode change. For instance, starting a carpool and splitting fuel cost could cut expenses in half, whereas it would be difficult to achieve $50 \%$ fuel savings just by driving more efficiently.

\section{ASSESSING VARIOUS DRIVER FEEDBACK APPROACHES}

The approach assessment involved applying the project insights described in the previous two sections to consider whether a given approach could work (e.g., if it effectively conveys appropriate information and instruction) and whether it would likely trigger significant behavior changes (e.g., if it is easy to use, avoids unintended consequences, and trumps other behavior influences). The observations about a number of different feedback approaches are summarized below.

- General Advice Sources - readily accessible but do not tailor feedback based on someone's actual driving behavior. They have the potential for a moderate overall impact.
- Driver Training Courses - unlikely to be attended by large numbers of drivers. They provide feedback on actual driving behavior, but because of limited participation their overall impact is expected to be low.

- Conventional Dashboards - many new vehicles provide both instantaneous and average fuel economy readouts. These displays have the potential to reach large numbers of people, but in the absence of accompanying feedback advice (and given that many vehicles in the legacy fleet have no such display) the overall impact would be moderate at best.

- Hybrid Vehicle Dashboards - typically have robust feedback displays conveniently accessible by the driver. Hybrid powertrains also alleviate some cycle-related efficiency concerns (such as through use of engine shut off and regenerative braking during stop-and-go driving), though at a cost premium. The combined technology and feedback impact for individual hybrid owners will be very large, but the aggregate impact remains limited due to relatively low hybrid vehicle penetration (both in new sales and in the existing vehicle fleet).

- Smart Phone Applications - have robust feedback, may have a competitive theme and can be adapted to existing vehicles. However, the barriers to use are significant-requiring availability of a smart phone, acquisition of software, purchase and installation of a vehicle mounting device, and use of the phone for driver feedback rather than other functions. Impact is expected to be moderate at best due to limited adoption rates.

- GPS Navigation Systems - some recent systems have driver feedback functionality built in. Without an accelerometer, though, the feedback is low-fidelity. As with smart phone applications, users must purchase and install the device. Expected impact is low.

- Offline Feedback Systems - largely limited to fleet users and require professional hardware installation. They do provide robust feedback and have the added advantage of not requiring driver attention. Because fleets may have more influence over their drivers' behavior, the impacts for fleets that use this approach may be significant.

- Dedicated Aftermarket Feedback Devices - These are generally dashboard-mounted devices with a wired or wireless connection to the vehicle's OBD port. The OBD connection provides the device with a high-quality data feed, including fuel flow rate, engine load, and vehicle speed. This allows the device to present throttle intensity as a surrogate for acceleration without the need for an accelerometer and the associated calibration requirements. However, these devices tend to be costly (on the order of \$200) and still require installation and setup, which will tend to limit their overall penetration and impact.

- Haptic Pedal Feedback - In this approach, driver feedback is provided by means of a vibrating accelerator pedal. When the 
driver accelerates at a rate greater than what the on-board computer deems efficient, the accelerator pedal vibrates to notify the driver to accelerate more gently. This approach would need to be built into the vehicle and would have similar advantages to manufacturer-integrated dashboard feedback. While manufacturers have researched this approach, it has not been incorporated into production vehicles, perhaps due to consumer acceptance concerns.

\section{Assessment Highlights}

The assessment identified seamless integration of comprehensive feedback into the manufacturer instrument cluster as one of the most promising approaches. Unfortunately, of vehicles on the road today, only hybrid and other advanced-technology vehicles seem to provide such comprehensive and seamlessly integrated feedback. (This perhaps reflects the heightened efficiency awareness of consumers in the hybrid vehicle market segment.) Some conventional vehicles do display instantaneous fuel economy, but few provide rigorous coaching on how to improve it. Many vehicles do not even provide this level of feedback, which obviously hinders the benefit that dashboard-integrated approaches can deliver to the existing vehicle fleet.

The most promising aftermarket approaches include getting feedback from an application or "app" on a driver's existing smart phone and/or using a dedicated device that incorporates information from the vehicle's OBD port. Regarding the quality of feedback information and instruction, OBD connection provides a slight advantage from data inputs such as vehicle-reported fuel rate and idle time. However, speed/ acceleration data either from the OBD or from the GPS/ accelerometer on a non-connected smart phone can provide the feedback basis for the most critical fuel efficiency behaviors.

With respect to adoption barriers, these two aftermarket approaches do not fare as well. Both require mounting a device in the vehicle such that the information display is visible but does not distract the driver. An OBD connection requires purchasing a dedicated device to obtain the vehicle data. The app approach gets around the expensive device purchase requirement for those who already own a smart phone, but requires users to firmly mount and calibrate the device (to get quality accelerometer data) and to give up other uses of the phone while driving (such as making a phone call, listening to music or getting directions). These barriers are not insurmountable, but can be significant for people who may not be enthusiastic about changing their driving style in the first place.

\section{CONCLUSIONS AND RECOMMENDATIONS}

\section{SUMMARY FINDINGS}

This study has shown that adopting efficient driving behaviors can result in fuel savings on the order of $20 \%$ for aggressively driven trips. Even starting from more moderate driving styles, efficient behaviors can reduce fuel consumption by $5 \%-10 \%$. Wide-spread penetration of such efficiency improvements could result in significant aggregate fuel savings. However, unlike efficiency technologies inherently integrated into a vehicle, realizing such aggregate fuel savings requires first motivating drivers to change how they drive. Important driver behavior influences include the actions of surrounding vehicles, the general flow of traffic, anxiety over trying to get somewhere quickly, and the power/ torque available from the vehicle. For many drivers, the perceived value of a fractional reduction in their fuel budget may be insufficient (relative to these other influences) to trigger them to make a concerted behavior change.

For individuals who are willing to change how they drive, the feedback mechanism they turn to for guidance will need to give them effective instruction on how to drive more efficiently and provide them useful reference point information (such as current fuel economy, acceleration rate and/or vehicle speed). Other important considerations for a feedback approach include its ease of use and how well it avoids unintended consequences that could add to a driver's reasons not to bother with the effort. Maximizing aggregate fuel savings requires feedback techniques that minimize adoption rate limitations and that push per-vehicle fuel savings towards the outer boundary of what an individual can achieve.

\section{RECOMMENDATIONS TO MAXIMIZE FUEL SAVINGS}

\section{Leverage Applications with Enhanced Incentives}

Commercial vehicle fleets present one application where fuel savings motivation can significantly outweigh the influences that work against behavior change in personal vehicles. Commercial vehicles tend to use a lot of fuel, so fleet managers strongly encourage their drivers to adopt efficient behaviors. Fleets may in many cases already implement some sort of driver training and incentive program. Working with such a motivated segment of drivers could allow larger scale refinement and evaluation of particular feedback techniques, leading to further deployment of the best fuel saving approach or approaches.

Getting large numbers of people to drive their personal vehicles more efficiently could also be accomplished through increasing their incentive to do so. One way to achieve this 
1. Watch the road, obey the law and drive safely (contributing to an accident will NOT save fuel).

2. Avoid speeds below $\approx 20 \mathrm{mph}$ and above $\approx 60 \mathrm{mph}$ (mpg progressively worsens in these regions).

3. Hold speed at steady in the $25-55 \mathrm{mph}$ range (e.g., keep centered on or between the color bars).

4. Slow down by letting off on the gas rather than by using the brake, and do so early to minimize time at very low speeds.

5. Above $10 \mathrm{mph}$, accelerate slowly (so that at least $2-3 \mathrm{sec}$ passes for every $10 \mathrm{mph}$ increase in speed).

6. Turn off engine when parked (do not idle).

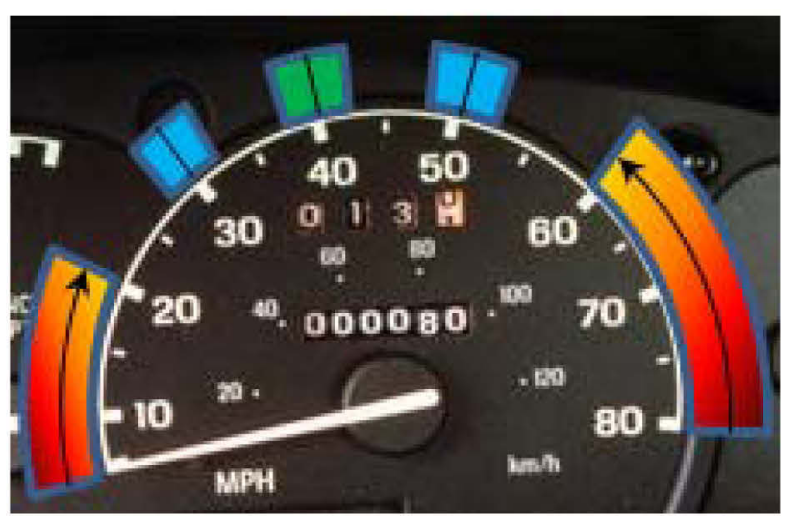

Figure 6. Example general advice with simple accompanying in-vehicle reference tool

could be to work collaboratively with insurance companies that are beginning to implement usage-based insurance [19]. The principle behind usage-based insurance is that insurance companies can better assess risk if they have direct measurements of things like distance driven and frequency of high speeds and accelerations. The companies are willing to give policyholders a discount on their premiums in exchange for installing a device in their vehicle that can provide this information. Because these same factors that contribute to insurance risk also increase fuel use, a single device could be enhanced to monitor as well as give feedback on improving such behaviors. Drivers could then realize a double benefit from adjusting their driving style: reducing their expenses for fuel as well as for auto insurance.

\section{Prepare a Simple and Widely Deployable}

\section{Approach}

Many people may remain reluctant to change driving habits as long as fuel prices hold steady (in order, for instance, to avoid potential angry honks or scowls from others for driving or accelerating too slowly). However, recent history illustrates the potential for gasoline prices to rise year after year by $10 \%-20 \%$ [20] and for this to trigger significantly increased public interest in saving fuel [12]. Gasoline prices may soon resume their upward rise, which could create a receptive environment for large deployment of an easy and straightforward driving efficiency tool.

The authors devised the simple concept described below while considering how to implement the project findings and the benefits of various existing approaches into an understandable and unobtrusive efficiency guide [21]. The general principle behind the proposed method is that an interested driver can learn the basic tenets of efficient driving from a short set of instructions, but having real-time reference information would help the driver really understand how to implement efficiency techniques. This can be accomplished with aftermarket devices or manufacturer-integrated feedback displays, albeit with limited penetration potential as discussed earlier. However, all vehicles contain a basic set of status gauges (speedometer, tachometer, etc.) that could be used to provide the most important driving efficiency reference information. Simply adding "landmarks" to those gauges would provide drivers with distinct vehicle operation reference points to help implement efficient driving instructions.

Figure 6 shows an example of how a simple set of instructions combined with speedometer enhancements could provide driving efficiency feedback. This concept could accomplish with inexpensive stickers on an existing analog gauge what would otherwise require a legacy vehicle owner to re-purpose a smart phone or purchase and mount another expensive device. Ideally, a website (also viewable by smart phone) could expand upon the six listed tips and encourage driver interaction after refueling in order to track progress. Note how the focus to remain between roughly $20-60 \mathrm{mph}$ and to minimize speed fluctuation (keeping acceleration low) aligns with the most fuel-efficient trips plotted in Figure 5.

\section{Make It Increasingly Automatic}

Another way to address the challenges of driver motivation and to increase adoption of efficient behavior is to give the vehicle more responsibility. New vehicles increasingly include safety and convenience features such as lane keep assist, adaptive cruise control, and early brake application for imminent collision avoidance. The technologies to produce these features could be used to create "green driving assist" in which the vehicle intelligently selects optimal acceleration/ deceleration rates and cruising speeds. The driver's full attention could then remain on the road to ensure safe operation (rather than occasionally diverting attention to a feedback device).

Over the past 25 years, research driven by highway safety, capacity improvement, and defense applications has dramatically advanced intelligent vehicle technologies toward even further levels of automated control. Google recently installed some of the developed component technologies in several vehicles, which have since autonomously driven 
thousands of miles on California roads [22]. An Italian company has similarly retrofitted vehicles and last year demonstrated an autonomous drive from Rome, Italy, to Shanghai, China [23] .

Continued autonomous driving advancements with an energy-efficiency focus could enable fuel savings to approach those this project calculated for complete drive profile optimization. Those savings exceeded $30 \%-40 \%$ over a variety of drive cycles with no changes to the vehicle. Another benefit of automation is that rather than having to convince drivers to change, people may instead demand the technology (regardless of fuel price) for value additions such as increased convenience and productivity, and reduced accidents and time spent in congestion. The improved safety, traffic flow, and guidance aspects could also enable compounding benefits from reducing vehicle weight and powertrain size, or even connecting vehicles to electric power while driving-each of which has been shown to deliver dramatic additional fuel savings.

\section{REFERENCES}

1. Vehicle fuel economy data, including official rating by the Environmental Protection Agency (EPA) and reported observations by individual drivers, posted on www.fueleconomy.gov. Accessed December 2011.

2. Earleywine, M.; Gonder, J.; Markel, T.; Thornton, M. (September 2010). "Simulated Fuel Economy and Performance of Advanced Hybrid Electric and Plug-in Hybrid Electric Vehicles Using In-Use Travel Profiles." Proceedings of the $6^{\text {th }}$ IEEE Vehicle Power and Propulsion Conference (VPPC). Lille, France.

3. Ojah, M.; Pearson, D. (August 2008). "2006 Austin/San Antonio GPS-Enhanced Household Travel Survey." Texas Transportation Institute.

4. O'Keefe, M., Simpson, A., Kelly, K., and Pedersen, D., "Duty Cycle Characterization and Evaluation Towards Heavy Hybrid Vehicle Applications," SAE Technical Paper 2007-01-0302, 2007, doi:10.4271/2007-01-0302.

5. Barkenbus, J. N. (2010). "Eco-driving: An Overlooked Climate Change Initiative." Energy Policy (38:2); pp. 762-769.

6. van der Voort, M.; Dougherty, M. S.; van Maarseveen, M. (2001). "A Prototype Fuel-Efficiency Support Tool."

Transportation Research Part C: Emerging Technologies (9:4); pp. 279-296.

7. Evans, L. (1979). "Driver Behavior Effects on Fuel Consumption in Urban Driving." Human Factors: The Journal of the Human Factors and Ergonomics Society (21:4); pp. 389-398.

8. Wilde, G. J. (1976). "Social Interaction Patterns in Driver Behavior: An Introductory Review." Human Factors: The
Journal of the Human Factors and Ergonomics Society (18:5); pp. 477-492.

9. Ehrhardt-Martinez, K. (April 2010). "Policy Innovations and People: Active Participants in the Energy Revolution." Prepared for the ACEEE 30th Anniversary Symposium: Energy and the Economic Imperative, April 2010. Washington, D.C.

10. Allcot, H. (October 2009). "Social Norms and Energy Conservation." Prepared for the Center for Energy and Environmental Policy Research, Massachusetts Institute of Technology.

11. Turrentine, T. S.; Kurani, K. S. (2007). "Car Buyers and Fuel Economy?” Energy Policy (35); pp. 1213-1223.

12. Consumer Reports. (August 2009). "Car Buyers are Thinking American, with Ford, GM Up; Chrysler Down." Accessed January 2011, from www.consumerreports.org.

13. J.D. Power Asia Pacific. (September 2010). "Advising Vehicle Owners on How to Improve Fuel Economy Has a Notable Positive Impact On Satisfaction with the NewVehicle Sales Process."

14. Boriboonsomsin, K.; Vu, A.; Barth, M. (2010). EcoDriving: Pilot Evaluation of Driving Behavior Changes. Riverside, CA: College of Engineering-Center for Environmental Research and Technology, University of California Riverside.

15. Graving, J. S.; Rakauskas, M. E.; Manser, M. P.; Jenness, J. W. (2010). "A Binary Response Method to Determine the Usability of Seven In-Vehicle Fuel Economy Displays." Proceedings of the Human Factors and Ergonomics Society 54th Annual Meeting. pp. 1546-1550.

16. Young, K.; Regan, M. (2007). Driver distraction: A review of the literature. Sydney, NSW: Australasian College of Road Safety.

17. Welk, D., "Mitigating Driver Distraction with Smart Phone Connectivity," Presentation from the SAE International "The Winning Path to Connected Vehicle Solutions," Telephone Webcast, January, 2011.

18. U.S. Environmental Protection Agency. (November 2010). "Light-Duty Automotive Technology, Carbon Dioxide Emissions, and Fuel Economy Trends: 1975 Through 2010." Technical Report EPA-420-R-10-023. http://www.epa.gov/ otaq/cert/mpg/fetrends/420r10023.pdf; pp. 5-34.

19. Progressive Insurance. (February 2011). "Snapshot Auto Insurance Discount." Company website, http:// www.progressive.com/snapshot/. Accessed February 22, 2011.

20. Energy Information Administration, EIA. (February 2011). "Petroleum and Other Liquids-Data on Prices." Agency website, http://www.eia.doe.gov/petroleum/data.cfm. Accessed February 16, 2011. 
21. Gonder, J. (February 2011). "Simple training aid for fuel economy improvement driver coaching." NREL Record of Invention No. ROI-11-00049.

22. Markoff, J. (October 2010). "Google Cars Drive Themselves, in Traffic." New York Times Article, http:// www.nytimes.com/2010/10/10/science/10google.html. Accessed February 20, 2011.

23. VisLab (October 2010). "VisLab's challenge successfully accomplished: the autonomous vehicles reached Shanghai Expo." Press release, http://vislab.it/media/press/21-VisLab \%20-\%2021-VIAC-done.pdf. Accessed February 20, 2011.

\section{CONTACT INFORMATION}

Jeffrey Gonder can be reached by e-mail at jeff.gonder@nrel.gov.

\section{ACKNOWLEDGMENTS}

The authors gratefully acknowledge the programmatic support of the U.S. Department of Energy, Office of Energy Efficiency and Renewable Energy, Vehicle Technologies Program, and wish to particularly thank Dr. Yury Kalish for his guidance and support. The authors also express their gratitude to Mekayla Beaver of Gloworm Insights and Dr. Barbara Farhar from the University of Colorado Institute of Behavioral Science for their advice on exploring the human factors and social science issues regarding driver influences and feedback receptiveness.

The Engineering Meetings Board has approved this paper for publication. It has successfully completed SAE's peer review process under the supervision of the session organizer. This process requires a minimum of three (3) reviews by industry experts. ISSN 0148-7191
Positions and opinions advanced in this paper are those of the author(s) and not necessarily those of SAE. The author is solely responsible for the content of the paper.

SAE Customer Service:

Tel: 877-606-7323 (inside USA and Canada)

Tel: 724-776-4970 (outside USA)

Fax: 724-776-0790

Email: CustomerService@sae.org

SAE Web Address: http://www.sae.org

Printed in USA 